Hrytsak Myroslava V., Popovych Daria V., Badiuk Nataliya S., Hrytsan Ivanna I., Zukow Walery. Similar neuroendocrine and metabolic effects of sulfate-chloride sodium-magnesium mineral waters "Myroslava" and "Khrystyna" of Truskavets' spa in healthy female rats. Journal of Education, Health and Sport. 2021;11(6):320-334. eISSN 2391-8306. DOI http://dx.doi.org/10.12775/JEHS.2021.11.06.035

https://apcz.umk.pl/czasopisma/index.php/JEHS/article/view/JEHS.2021.11.06.035

https://zenodo.org/record/5818119

The journal has had 5 points in Ministry of Science and Higher Education parametric evaluation. § 8. 2) and § 12. 1. 2) 22.02.2019. @ The Authors 2021; This article is published with open access at Licensee Open Journal Systems of Nicolaus Copernicus University in Torun, Poland Open Access. This article is distributed under the terms of the Creative Commons Attribution Noncommercial License which permits any Attribution Non commercial license Share alike. (http://creativecommons.org/licenses/by-nc-sa/4.0/) which permits unrestricted, non commercial use, distribution and reproduction in any medium, provided the work is properly cited.
is no conflict of interests regarding the publication of this paper.

Received: 15.05.2021. Revised: 21.05.2021. Accepted: 29.06.2021.

\title{
SIMILAR NEUROENDOCRINE AND METABOLIC EFFECTS OF SULFATE- CHLORIDE SODIUM-MAGNESIUM MINERAL WATERS "MYROSLAVA" AND "KHRYSTYNA" OF TRUSKAVETS' SPA IN HEALTHY FEMALE RATS
}

\author{
Myroslava V. Hrytsak ${ }^{1,2}$, Daria V. Popovych ${ }^{3}$, Nataliya S. Badiuk ${ }^{1,4}$, \\ Ivanna I. Hrytsan ${ }^{1,4}$, Walery Zukow ${ }^{5}$
}

1SE Ukrainian Research Institute for Medicine of Transport, Odesa badiuk ns@ukr.net ${ }^{2}$ Scientific group of Balneology of Hotel\&Spa Complex "Karpaty", Truskavets', Ukraine ira barschyk@ukr.net hrytsak.myroslava@gmail.com ${ }^{3}$ IY Horbachevs'kyi National Medical University, Ternopil', Ukraine darakoz@yahoo.com

${ }^{4}$ International Medical University, Odesa, Ukraine ${ }^{5}$ Nicolaus Copernicus University, Torun, Poland w.zukow@wp.pl

Background. In order to expand the hydro-mineral base of Truskavets' spa by diluting brine $(130 \mathrm{~g} / \mathrm{L})$, two new sulphate-chloride sodium-magnesium mineral waters "Myroslava" (5 g/L) and "Khrystyna" (10 g/L) were created. This report is the first in a series of experimental studies of their physiological activity in line with the concepts of neuroendocrine-immune complex and functional-metabolic continuum. Materials and Methods. Experiment was performed on 50 healthy female Wistar rats 220-300 g divided into 4 groups. Animals of the first group remained intact, using tap water from drinking ad libitum. Rats of the control group for 6 days administered a single tap water through the tube at a dose of 1,5 mL/100 $\mathrm{g}$ of body mass. The rats of the main groups received the water "Myroslava" and "Khrystyna". The day after the completion of the drinking course in all rats assessed the state of autonomous regulation by parameters of the HRV. The plasma levels of the hormones of adaptation were determined: corticosterone, triiodothyronine and testosterone (by the ELISA); as well as electrolytes, nitric metabolites, lipid peroxidation products and antioxidant enzymes as well as cholesterol, glucose, amylase and middle mass molecules. Most of the listed parameters of metabolism as well as 17-ketosteroids were determined in daily urine. In the adrenal glands the thickness of glomerular, fascicular, reticular and medullar zones was measured. Results. To identify exactly those parameters, the set of which three groups of animals differ significantly from each other, the information field of the registered parameters was subjected to discriminant analysis. The program included in the model 6 endocrine and 11 metabolic parameters, as well as glomerular filtration. Conclusion. The newly created sulfate-chloride sodium-magnesium drinking mineral waters of Truskavets' spa have similar neuroendocrine and metabolic effects on healthy old female rats significantly different from daily water. 
Keywords: sulfate-chloride sodium-magnesium drinking mineral waters, Truskavets' spa, neuroendocrine and metabolic parameters, female rats.

\section{INRODUCTION}

In order to expand the hydro-mineral base of Truskavets' spa by diluting brine $(130 \mathrm{~g} / \mathrm{L})$, two new sulphate-chloride sodium-magnesium mineral waters "Myroslava" (5 g/L) and "Khrystyna" (10 g/L) were created. This report is the first in a series of experimental studies of their physiological activity in line with the concepts of neuroendocrine-immune complex [14,18,21-23,26] and functional-metabolic continuum [8].

\section{MATERIALS AND METHODS}

Experiment was performed on 50 healthy old female Wistar rats 220-300 g $(\mathrm{M} \pm \mathrm{SD}=262 \pm 23 \mathrm{~g})$ divided into 4 groups. Animals of the first group (10) remained intact, using tap water from drinking ad libitum. Rats of the second (control) group (10) for 6 days administered a single tap water through the tube at a dose of $1,5 \mathrm{~mL} / 100 \mathrm{~g}$ of body mass. The rats of the main groups received the water "Myroslava" (15) and "Khrystyna" (15), prepared from the brine of the 27- $K$ well of the Truskavetsian field by appropriate dilutions with fresh water. The chemical composition of the applied waters (as well as, for comparison, the "Sofia" water of the Truskavets' spa), according to the Truskavetsian Hydrogeological Regime-operational station, is given in Table 1.

Table 1. Chemical composition of fresh and mineral waters

\begin{tabular}{|l|c|c|c|c|}
\hline & $\begin{array}{l}\text { Daily } \\
\text { Water }\end{array}$ & Sofiya & Khrystyna & Myroslava \\
\hline \multicolumn{5}{|c|}{ Electrolytes, $\mathbf{~ M M / L ~}$} \\
\hline $\mathbf{S O}_{4}{ }^{2-}$ & 1,2 & 13,1 & $\mathbf{5 4 , 5}$ & $\mathbf{2 7 , 3}$ \\
\hline $\mathbf{C l}^{-}$ & 3,4 & 142 & $\mathbf{4 3}$ & $\mathbf{2 2}$ \\
\hline $\mathbf{N a}^{+}$ & 0,5 & 156 & $\mathbf{1 2 7}$ & $\mathbf{6 4}$ \\
\hline $\mathbf{M g}^{2+}$ & 0,5 & 4,3 & $\mathbf{1 1 , 9}$ & $\mathbf{6 , 0}$ \\
\hline $\mathrm{Ca}^{2+}$ & 3,4 & 5,3 & 0,77 & 0,39 \\
\hline $\mathrm{HCO}_{3}{ }^{-}$ & 2,9 & 7,5 & 0,6 & 0,3 \\
\hline $\mathrm{K}^{+}$ & 0,4 & 0,3 & 0,4 & 0,2 \\
\hline \multicolumn{5}{|c|}{ Trace elements, $\mathbf{~ m g / L}$} \\
\hline $\mathrm{Br}^{-}$ & 8,3 & 6,7 & 2,68 & 1,34 \\
\hline $\mathrm{F}^{-}$ & 0,95 & 0,52 & 1,16 & 0,58 \\
\hline $\mathrm{H}_{2} \mathrm{SiO}_{3}$ & 5 & 4,43 & 0,13 & 0,065 \\
\hline $\mathrm{H}_{3} \mathrm{BO}_{3}$ & 0,25 & 8,39 & 0,10 & 0,05 \\
\hline $\mathrm{J}^{-}$ & 0,025 & 1,29 & 0,004 & 0,002 \\
\hline $\mathrm{C}_{\text {organ }}$ & 5,0 & 5,5 & 0,83 & 0,42 \\
\hline
\end{tabular}

The day after the completion of the drinking course in all rats, at first, assessed the state of autonomous regulation. For this purpose, under an easy ether anesthesia, for 15-20 sec ECG was recorded in the lead II, inserting needle electrodes under the skin of the legs, followed by the calculation of the parameters of the HRV: mode (Mo), amplitude of the mode (AMo) and variational swing (MxDMn) as markers of the humoral channel of regulation, sympathetic and vagal tones respectively [2].

Animals were then placed in individual chambers with perforated bottom for collecting daily urine.

The experiment was completed by decapitation of rats in order to collect as much blood as possible. 
The plasma levels of the hormones of adaptation were determined: corticosterone, triiodothyronine and testosterone (by the ELISA); as well as electrolytes: calcium (by reaction with arsenase III), magnesium (by reaction with colgamite), phosphates (phosphatemolybdate method), chloride (mercury-rhodanidine method), sodium and potassium (both in plasma and in erythrocytes) by flamming photometry; nitric metabolites: creatinine (by Jaffe's color reaction by Popper's method), urea (urease method by reaction with phenolhypochlorite), uric acid (uricase method), medium molecular polypeptides (by spectrophotometric method), bilirubin (by diazoreaction using the Jedrashik-Kleghorn-Grof method) [7]; lipid peroxidation products: diene conjugates (spectrophotometry of the heptane phase of the lipids extract [6]) and malondyaldehide (in the test with thiobarbituric acid [1]), antioxidant enzymes: superoxide dismutase erythrocytes (according to the degree of inhibition of reduction of nitroblue tetrazolium in the presence of N-methylphenazonium metasulphate and NADH $[4,15]$ ) and catalase plasma (at the rate of decomposition of hydrogen peroxide [13]), as well as cholesterol (by a direct method after the classic reaction by Zlatkis-Zack), amylase (Karavay's amyloclastic method with starch substrate) and glucose (glucose-oxidase method) [7].

Most of the listed parameters of metabolism were also determined in daily urine. The latter also determined the concentration of 17-ketosteroids (by color reaction with $\mathrm{m}$ dinitrobenzene). By the size of the diuresis and the level of creatinine in plasma and urine, glomerular filtration and tubular reabsorption were calculated.

According to the parameters of electrolyte exchange, hormonal activity was evaluated: parathyroid by coefficients $(\mathrm{Cap} / \mathrm{Pp})^{0,5}$ and $(\mathrm{Pu} / \mathrm{Cau})^{0,5}$, calcitonin by coefficients $(\mathrm{Cap} \cdot \mathrm{Pp})^{-0,5}$ and $(\mathrm{Cau} \bullet \mathrm{Pu})^{0,5}$ as well as mineralocorticoid by coefficients $(\mathrm{Nap} / \mathrm{Kp})^{0,5}$ and $(\mathrm{Ku} / \mathrm{Nau})^{0,5}$, based on their classical effects and recommendations by Popovych IL [23].

Urine lithogenicity index (Lith) was also calculated by the Tiselius' HS [24] formula modifed by Flyunt VR et al [5]:

Lith $=(\text { Uric acid } \bullet \text { Calcium/Magnesium } \bullet \text { Creatinine })^{0,25}$.

The analyzes were carried out according to the instructions. The analyzers "Tecan" (Oesterreich), "Pointe-180" ("Scientific", USA) and "Reflotron" (Boehringer Mannheim, BRD) were used with appropriate sets and a flaming spectrophotometer "CФ-47".

After decapitation, the adrenal glands were removed and weighed, then the thickness of glomerular, fascicular, reticular and medullar zones was measured under a microscope [3].

Digital material is statistically processed on a computer using the software package "Statistica 64".

\section{RESULTS AND DISCUSSION}

This article will look at the neuroendocrine and metabolic effects common to both mineral waters, so the rats they load are grouped together in the "Salt Waters" group. The specific effects of waters will be the subject of the next study, theses of which were published earlier [9]. To identify exactly those parameters, the set of which three groups of animals differ significantly from each other, the information field of the registered parameters was subjected to discriminant analysis [12]. The program forward stepwise included in the model 6 endocrine and 11 metabolic parameters, including 7 electrolytes of plasma and urine and 4 non-electrolytes of plasma and urine, as well as glomerular filtration (Tables 2 and 7). The rest of the registered parameters were outside the discriminant model (Tables 3-6). 
Table 2. Discriminant Function Analysis Summary

Step 18, N of Variables currently in the model: 18; Grouping: 3 groups

Wilks' Lambda: 0,1058; approx. $\mathrm{F}_{(37)}=3,46 ; \mathrm{p}<10^{-5}$

\begin{tabular}{|c|c|c|c|c|c|c|c|c|}
\hline \multirow[b]{2}{*}{$\begin{array}{l}\text { Variables } \\
\text { currently in the model }\end{array}$} & \multicolumn{3}{|c|}{ Groups (n) } & \multicolumn{5}{|c|}{ Parameters of Wilks' Statistics } \\
\hline & $\begin{array}{l}\text { Intact } \\
\text { rats } \\
(10)\end{array}$ & $\begin{array}{l}\text { Daily } \\
\text { Water } \\
\text { (10) }\end{array}$ & $\begin{array}{l}\text { Salt } \\
\text { Waters } \\
\text { (30) }\end{array}$ & $\begin{array}{l}\text { Wil } \\
\text { ks' } \\
\Lambda\end{array}$ & $\begin{array}{l}\text { Par- } \\
\text { tial } \\
\Lambda\end{array}$ & $\begin{array}{l}\text { F-re- } \\
\text { move } \\
(2,30)\end{array}$ & $\begin{array}{l}\mathrm{p}- \\
\text { level }\end{array}$ & $\begin{array}{l}\text { Tole- } \\
\text { rancy }\end{array}$ \\
\hline $\begin{array}{l}\text { Calcium } \\
\text { Plasma, } \\
\mathrm{mM} / \mathrm{L}\end{array}$ & $\begin{array}{l}3,35 \\
1 \\
0\end{array}$ & $\begin{array}{l}2,08 \\
0,62 \\
-1,24\end{array}$ & $\begin{array}{l}2,71 \\
0,81 \\
-0,63\end{array}$ & 0,110 & 0,964 & 0,56 & 0,579 & 0,437 \\
\hline $\begin{array}{l}\text { Potassium } \\
\text { Plasma, } \\
\mathrm{mM} / \mathrm{L}\end{array}$ & $\begin{array}{l}4,23 \\
1 \\
0 \\
\end{array}$ & $\begin{array}{l}3,54 \\
0,84 \\
-0,98 \\
\end{array}$ & $\begin{array}{l}3,38 \\
0,80 \\
-1,21 \\
\end{array}$ & 0,157 & 0,673 & 7,28 & 0,003 & 0,355 \\
\hline $\begin{array}{l}\text { Sodium } \\
\text { Excretion, } \\
\mu \mathrm{M} / 24 \mathrm{~h} \cdot 100 \mathrm{~g} \text { Body Mass }\end{array}$ & $\begin{array}{l}135 \\
1 \\
0 \\
\end{array}$ & $\begin{array}{l}76 \\
0,56 \\
-0,70 \\
\end{array}$ & $\begin{array}{l}219 \\
1,63 \\
+1,00 \\
\end{array}$ & 0,118 & 0,897 & 1,72 & 0,196 & 0,366 \\
\hline $\begin{array}{l}(\mathrm{Cap} / \mathrm{Pp})^{0,5} \text { as Parathyroid } \\
\text { Activity }\end{array}$ & $\begin{array}{l}2,56 \\
1 \\
0 \\
\end{array}$ & $\begin{array}{l}1,58 \\
0,62 \\
-0,84 \\
\end{array}$ & $\begin{array}{l}1,83 \\
0,71 \\
-0,63 \\
\end{array}$ & 0,150 & 0,706 & 6,25 & 0,005 & 0,127 \\
\hline $\begin{array}{l}\text { Glomerular } \\
\text { Filtration, } \\
\mu \mathrm{L} / \min \bullet 100 \mathrm{~g} \text { Body Mass }\end{array}$ & $\begin{array}{l}86,0 \\
1 \\
0\end{array}$ & $\begin{array}{l}85,2 \\
0,99 \\
-0,03\end{array}$ & $\begin{array}{l}146,7 \\
1,71 \\
+1,97\end{array}$ & 0,115 & 0,922 & 1,27 & 0,296 & 0,613 \\
\hline $\begin{array}{l}\text { Glomerular Zone } \\
\text { of Adrenal Cortex, } \\
\mu \mathrm{M}\end{array}$ & $\begin{array}{l}193 \\
1 \\
0 \\
\end{array}$ & $\begin{array}{l}207 \\
1,07 \\
+0,29 \\
\end{array}$ & $\begin{array}{l}184 \\
0,95 \\
-0,21 \\
\end{array}$ & 0,120 & 0,881 & 2,02 & 0,151 & 0,484 \\
\hline $\begin{array}{l}\text { Katalase Activity } \\
\text { Plasma, } \\
\mu \mathrm{M} / \mathrm{h} \cdot \mathrm{L}\end{array}$ & $\begin{array}{l}103 \\
1 \\
0 \\
\end{array}$ & $\begin{array}{l}148 \\
1,43 \\
+1,58 \\
\end{array}$ & $\begin{array}{l}125 \\
1,21 \\
+0,77 \\
\end{array}$ & 0,138 & 0,769 & 4,50 & 0,019 & 0,138 \\
\hline $\begin{array}{l}\text { Mode HRV } \\
\text { as Humoral channel, } \\
\text { msec }\end{array}$ & $\begin{array}{l}124 \\
1 \\
0\end{array}$ & $\begin{array}{l}105 \\
0,85 \\
-1,27\end{array}$ & $\begin{array}{l}119 \\
0,96 \\
-0,34\end{array}$ & 0,133 & 0,795 & 3,87 & 0,032 & 0,415 \\
\hline $\begin{array}{l}\text { Diene conjugates } \\
\text { Plasma, } \\
\mathbf{E}^{232} / \mathrm{mL}\end{array}$ & $\begin{array}{l}1,34 \\
1 \\
0 \\
\end{array}$ & $\begin{array}{l}1,42 \\
1,06 \\
+0,20 \\
\end{array}$ & $\begin{array}{l}1,50 \\
1,12 \\
+0,39 \\
\end{array}$ & 0,149 & 0,710 & 6,11 & 0,006 & 0,389 \\
\hline $\begin{array}{l}\text { Sodium } \\
\text { Plasma, } \\
\mathrm{mM} / \mathrm{L} \\
\end{array}$ & $\begin{array}{l}128,6 \\
1 \\
0 \\
\end{array}$ & $\begin{array}{l}131,9 \\
1,03 \\
+0,65 \\
\end{array}$ & $\begin{array}{l}127,7 \\
0,99 \\
-0,16 \\
\end{array}$ & 0,135 & 0,784 & 4,12 & 0,026 & 0,065 \\
\hline $\begin{array}{l}\text { Cholesterol } \\
\text { Plasma } \\
\mathrm{mM} / \mathrm{L}\end{array}$ & $\begin{array}{l}1,57 \\
1 \\
0\end{array}$ & $\begin{array}{l}1,70 \\
1,08 \\
+0,28\end{array}$ & $\begin{array}{l}1,57 \\
1,00 \\
-0,01\end{array}$ & 0,114 & 0,927 & 1,19 & 0,319 & 0,591 \\
\hline $\begin{array}{l}\text { Medullar } \\
\text { Zone of Adrenals, } \\
\mu \mathrm{M}\end{array}$ & $\begin{array}{l}94 \\
1 \\
0 \\
\end{array}$ & $\begin{array}{l}65 \\
0,69 \\
-0,93 \\
\end{array}$ & $\begin{array}{l}94 \\
1,00 \\
-0,01 \\
\end{array}$ & 0,124 & 0,855 & 2,55 & 0,095 & 0,366 \\
\hline $\begin{array}{l}\text { Triiodothyronine } \\
\text { Plasma, } \\
\text { nM/L }\end{array}$ & $\begin{array}{l}2,14 \\
1 \\
0\end{array}$ & $\begin{array}{l}2,11 \\
0,99 \\
-0,05\end{array}$ & $\begin{array}{l}2,35 \\
1,10 \\
+0,36 \\
\end{array}$ & 0,122 & 0,869 & 2,26 & 0,122 & 0,509 \\
\hline $\begin{array}{l}\text { Phosphate } \\
\text { Plasma, } \\
\mathrm{mM} / \mathrm{L}\end{array}$ & $\begin{array}{l}0,72 \\
1 \\
0 \\
\end{array}$ & $\begin{array}{l}1,01 \\
1,41 \\
+0,65 \\
\end{array}$ & $\begin{array}{l}0,96 \\
1,34 \\
+0,53 \\
\end{array}$ & 0,129 & 0,823 & 3,24 & 0,053 & 0,104 \\
\hline $\begin{array}{l}\text { Chloride } \\
\text { Plasma, } \\
\mathrm{mM} / \mathrm{L}\end{array}$ & $\begin{array}{l}94,3 \\
1 \\
0\end{array}$ & $\begin{array}{l}95,4 \\
1,01 \\
+0,14\end{array}$ & $\begin{array}{l}90,7 \\
0,96 \\
-0,51\end{array}$ & 0,120 & 0,882 & 2,00 & 0,153 & 0,061 \\
\hline $\begin{array}{l}\text { Katalase Activity } \\
\text { Urine, } \\
\mu \mathrm{M} / \mathrm{h} \cdot \mathrm{L}\end{array}$ & $\begin{array}{l}123 \\
1 \\
0\end{array}$ & $\begin{array}{l}149 \\
1,22 \\
+0,96\end{array}$ & $\begin{array}{l}146 \\
1,19 \\
+0,86\end{array}$ & 0,124 & 0,853 & 2,59 & 0,092 & 0,132 \\
\hline $\begin{array}{l}\text { Testosterone } \\
\text { Plasma, } \\
\text { nM/L }\end{array}$ & $\begin{array}{l}3,93 \\
1 \\
0 \\
\end{array}$ & $\begin{array}{l}6,04 \\
1,54 \\
+1,97 \\
\end{array}$ & $\begin{array}{l}4,75 \\
1,21 \\
+0,77 \\
\end{array}$ & 0,114 & 0,928 & 1,16 & 0,326 & 0,551 \\
\hline $\begin{array}{l}\text { Magnesium } \\
\text { Plasma, } \\
\mathrm{mM} / \mathrm{L}\end{array}$ & $\begin{array}{l}0,88 \\
1 \\
0 \\
\end{array}$ & $\begin{array}{l}0,99 \\
1,13 \\
+0,19 \\
\end{array}$ & $\begin{array}{l}0,73 \\
0,83 \\
-0,24 \\
\end{array}$ & 0,113 & 0,933 & 1,09 & 0,351 & 0,412 \\
\hline
\end{tabular}


Note. In each column, the first line is the average value, the second is the fraction of the norm, and the third is the Z-score.

Table 3. Discriminant Function Analysis Summary. Neuro-endocrine variables currently not in the model

\begin{tabular}{|c|c|c|c|c|c|c|c|c|}
\hline \multirow[b]{2}{*}{ Variables } & \multicolumn{3}{|c|}{ Groups (n) } & \multicolumn{5}{|c|}{ Parameters of Wilks' Statistics } \\
\hline & \begin{tabular}{|l|} 
Intact \\
rats \\
$(10)$ \\
\end{tabular} & \begin{tabular}{|l|} 
Daily \\
Water \\
$(\mathbf{1 0})$ \\
\end{tabular} & \begin{tabular}{|l|} 
Salt \\
Waters \\
$(30)$ \\
\end{tabular} & $\begin{array}{l}\text { Wilks' } \\
\Lambda\end{array}$ & $\begin{array}{l}\text { Parti- } \\
\text { al } \Lambda\end{array}$ & $\begin{array}{l}\mathrm{F} \text { to } \\
\text { en- } \\
\text { ter } \\
\end{array}$ & $\begin{array}{l}\text { p- } \\
\text { level }\end{array}$ & \begin{tabular}{|l|} 
Tole- \\
rancy
\end{tabular} \\
\hline $\begin{array}{l}\text { MxDMn HRV } \\
\text { as Vagal tone, } \\
\text { msec }\end{array}$ & \begin{tabular}{|l|}
53 \\
1 \\
0 \\
\end{tabular} & \begin{tabular}{|l|}
37 \\
0,70 \\
$-0,39$ \\
\end{tabular} & \begin{tabular}{|l|}
55 \\
1,04 \\
$+0,05$ \\
\end{tabular} & 0,100 & 0,948 & 0,79 & 0,463 & 0,179 \\
\hline $\begin{array}{l}\text { Amplitude Mode HRV } \\
\text { as Sympathetic tone, } \\
\%\end{array}$ & $\begin{array}{l}56 \\
1 \\
0\end{array}$ & \begin{tabular}{|l}
70 \\
1,26 \\
$+0,84$ \\
\end{tabular} & \begin{tabular}{|l|}
56 \\
1,00 \\
$-0,01$
\end{tabular} & 0,099 & 0,940 & 0,93 & 0,406 & 0,112 \\
\hline $\begin{array}{l}\text { Corticosterone } \\
\text { Plasma, } \\
\text { nM/L }\end{array}$ & \begin{tabular}{|l|}
482 \\
1 \\
0 \\
\end{tabular} & \begin{tabular}{|l|}
383 \\
0,80 \\
$-0,78$ \\
\end{tabular} & \begin{tabular}{|l|}
413 \\
0,86 \\
$-0,55$
\end{tabular} & 0,103 & 0,970 & 0,45 & 0,641 & 0,701 \\
\hline $\begin{array}{l}(\mathrm{Nap} / \mathrm{Kp})^{0,5} \\
\text { as Mineralocorticoid } \\
\text { Activity }\end{array}$ & \begin{tabular}{|l|}
5,57 \\
1 \\
0
\end{tabular} & \begin{tabular}{|l|}
6,22 \\
1,12 \\
$+1,18$
\end{tabular} & $\begin{array}{l}6,26 \\
1,12 \\
+1,25\end{array}$ & 0,105 & 0,988 & 0,18 & 0,839 & 0,038 \\
\hline $\begin{array}{l}(\mathrm{Ku} / \mathrm{Nau})^{0,5} \\
\text { as Mineralocorticoid } \\
\text { Activity }\end{array}$ & \begin{tabular}{|l|}
1,44 \\
1 \\
0 \\
\end{tabular} & \begin{tabular}{|l|}
2,34 \\
1,63 \\
$+1,09$ \\
\end{tabular} & $\begin{array}{l}1,39 \\
0,97 \\
-0,05 \\
\end{array}$ & 0,103 & 0,976 & 0,36 & 0,699 & 0,226 \\
\hline $\begin{array}{l}\text { 17-Ketosteroide } \\
\text { Excretion, } \\
\text { nM/24h } 100 \mathrm{~g} \text { Body Mass }\end{array}$ & \begin{tabular}{|l|}
61 \\
1 \\
0 \\
\end{tabular} & \begin{tabular}{|l|}
59 \\
0,97 \\
$-0,04$ \\
\end{tabular} & \begin{tabular}{|l|}
75 \\
1,22 \\
$+0,24$ \\
\end{tabular} & 0,104 & 0,986 & 0,20 & 0,817 & 0,453 \\
\hline $\begin{array}{l}\text { Adrenals } \\
\text { Mass Index, } \\
\text { mg/100 g Body Mass }\end{array}$ & $\begin{array}{l}25,2 \\
1 \\
0\end{array}$ & \begin{tabular}{|l|}
26,8 \\
1,06 \\
$+0,31$
\end{tabular} & \begin{tabular}{|l|}
26,1 \\
1,04 \\
$+0,18$
\end{tabular} & 0,105 & 0,990 & 0,15 & 0,863 & 0,842 \\
\hline $\begin{array}{l}\text { Fascicular } \\
\text { Zone of Adrenal Cortex, } \\
\mu \mathrm{M}\end{array}$ & \begin{tabular}{|l|}
391 \\
1 \\
0 \\
\end{tabular} & \begin{tabular}{|l|}
398 \\
1,02 \\
$+0,09$ \\
\end{tabular} & \begin{tabular}{|l|}
420 \\
1,08 \\
$+0,34$ \\
\end{tabular} & 0,104 & 0,983 & 0,25 & 0,778 & 0,483 \\
\hline $\begin{array}{l}\text { Reticular } \\
\text { Zone of Adrenal Cortex, } \\
\mu \mathrm{M}\end{array}$ & \begin{tabular}{|l|}
43 \\
1 \\
0
\end{tabular} & \begin{tabular}{|l|}
40 \\
0,95 \\
$-0,29$ \\
\end{tabular} & \begin{tabular}{|l|}
43 \\
1,01 \\
$+0,04$ \\
\end{tabular} & 0,101 & 0,958 & 0,63 & 0,540 & 0,614 \\
\hline $\begin{array}{l}(\text { Cap•Pp) })^{-0,5} \\
\text { as Calcitonin } \\
\text { Activity }\end{array}$ & \begin{tabular}{|l|}
0,79 \\
1 \\
0 \\
\end{tabular} & \begin{tabular}{|l|}
0,78 \\
0,98 \\
$-0,05$ \\
\end{tabular} & \begin{tabular}{|l|}
0,78 \\
0,92 \\
$-0,18$ \\
\end{tabular} & 0,105 & 0,994 & 0,08 & 0,918 & 0,034 \\
\hline $\begin{array}{l}(\mathrm{Cau} \cdot \mathrm{Pu})^{0,5} \\
\text { as Calcitonin } \\
\text { Activity }\end{array}$ & \begin{tabular}{|l|}
3,63 \\
1 \\
0
\end{tabular} & \begin{tabular}{|l|}
3,63 \\
1,00 \\
0,00
\end{tabular} & $\begin{array}{l}3,50 \\
0,97 \\
-0,15\end{array}$ & 0,103 & 0,973 & 0,41 & 0,668 & 0,582 \\
\hline $\begin{array}{l}(\mathrm{Pu} / \mathrm{Cau})^{0,5} \text { as } \\
\text { Parathyroid } \\
\text { Activity }\end{array}$ & $\begin{array}{l}1,76 \\
1 \\
0\end{array}$ & $\begin{array}{l}1,80 \\
1,02 \\
+0,08\end{array}$ & $\begin{array}{l}1,82 \\
1,03 \\
+0,13\end{array}$ & 0,102 & 0,966 & 0,51 & 0,605 & 0,527 \\
\hline
\end{tabular}


Table 4. Discriminant Function Analysis Summary. Urine and erythrocytes electrolytic variables currently not in the model

\begin{tabular}{|c|c|c|c|c|c|c|c|c|}
\hline \multirow[b]{2}{*}{ Variables } & \multicolumn{3}{|c|}{ Groups (n) } & \multicolumn{5}{|c|}{ Parameters of Wilks' Statistics } \\
\hline & $\begin{array}{l}\text { Intact } \\
\text { rats } \\
(10)\end{array}$ & \begin{tabular}{|l|} 
Daily \\
Water \\
$(\mathbf{1 0})$ \\
\end{tabular} & \begin{tabular}{|l|} 
Salt \\
Waters \\
$(30)$ \\
\end{tabular} & $\begin{array}{l}\text { Wilks' } \\
\Lambda\end{array}$ & $\begin{array}{l}\text { Parti- } \\
\text { al } \Lambda\end{array}$ & $\begin{array}{l}\mathrm{F} \text { to } \\
\text { en- } \\
\text { ter } \\
\end{array}$ & $\begin{array}{l}\text { p- } \\
\text { level }\end{array}$ & $\begin{array}{l}\text { Tole- } \\
\text { rancy }\end{array}$ \\
\hline $\begin{array}{l}\text { Magnesium } \\
\text { Urine, } \\
\mathrm{mM} / \mathrm{L}\end{array}$ & $\begin{array}{l}2,56 \\
1 \\
0\end{array}$ & \begin{tabular}{|l|}
2,34 \\
0,91 \\
$-0,12$ \\
\end{tabular} & \begin{tabular}{|l|}
2,69 \\
1,05 \\
$+0,07$ \\
\end{tabular} & 0,103 & 0,976 & 0,36 & 0,699 & 0,226 \\
\hline $\begin{array}{l}\text { Potassium } \\
\text { Urine, } \\
\mathrm{mM} / \mathrm{L}\end{array}$ & $\begin{array}{l}131 \\
1 \\
0\end{array}$ & \begin{tabular}{l|}
130 \\
0,99 \\
$-0,02$ \\
\end{tabular} & $\begin{array}{l}122 \\
0,93 \\
-0,23\end{array}$ & 0,103 & 0,976 & 0,36 & 0,699 & 0,226 \\
\hline $\begin{array}{l}\text { Calcium } \\
\text { Urine, } \\
\mathrm{mM} / \mathrm{L}\end{array}$ & $\begin{array}{l}2,10 \\
1 \\
0\end{array}$ & \begin{tabular}{|l|}
2,17 \\
1,03 \\
$+0,19$ \\
\end{tabular} & \begin{tabular}{|l|}
2,08 \\
0,99 \\
$-0,03$ \\
\end{tabular} & 0,104 & 0,986 & 0,20 & 0,817 & 0,453 \\
\hline $\begin{array}{l}\text { Phosphate } \\
\text { Urine, } \\
\text { mM/L }\end{array}$ & $\begin{array}{l}6,39 \\
1 \\
0\end{array}$ & \begin{tabular}{|l|}
6,20 \\
0,97 \\
$-0,24$ \\
\end{tabular} & $\begin{array}{l}6,13 \\
0,96 \\
-0,33 \\
\end{array}$ & 0,105 & 0,990 & 0,15 & 0,863 & 0,842 \\
\hline $\begin{array}{l}\text { Sodium } \\
\text { Urine, } \\
\mathrm{mM} / \mathrm{L}\end{array}$ & $\begin{array}{l}105 \\
1 \\
0 \\
\end{array}$ & \begin{tabular}{|l|}
55 \\
0,52 \\
$-0,76$ \\
\end{tabular} & \begin{tabular}{|l|}
126 \\
1,20 \\
$+0,32$ \\
\end{tabular} & 0,104 & 0,983 & 0,25 & 0,778 & 0,483 \\
\hline $\begin{array}{l}\text { Chloride } \\
\text { Urine, } \\
\mathrm{mM} / \mathrm{L}\end{array}$ & $\begin{array}{l}115 \\
1 \\
0\end{array}$ & \begin{tabular}{|l|}
70 \\
0,61 \\
$-0,56$
\end{tabular} & $\begin{array}{l}137 \\
1,19 \\
+0,28\end{array}$ & 0,101 & 0,958 & 0,63 & 0,540 & 0,614 \\
\hline $\begin{array}{l}\text { Phosphates } \\
\text { Excretion, } \\
\mu \mathrm{M} / 24 \mathrm{~h} \cdot 100 \mathrm{~g} \text { Body Mass }\end{array}$ & $\begin{array}{l}9,4 \\
1 \\
0 \\
\end{array}$ & \begin{tabular}{|l|}
9,9 \\
1,05 \\
$+0,08$ \\
\end{tabular} & \begin{tabular}{|l|}
11,5 \\
1,22 \\
$+0,33$ \\
\end{tabular} & 0,105 & 0,988 & 0,18 & 0,839 & 0,038 \\
\hline $\begin{array}{l}\text { Potassium } \\
\text { Excretion, } \\
\mu \mathrm{M} / 24 \mathrm{~h} \bullet 100 \mathrm{~g} \text { Body Mass }\end{array}$ & $\begin{array}{l}189 \\
1 \\
0 \\
\end{array}$ & \begin{tabular}{|l|}
203 \\
1,08 \\
$+0,12$ \\
\end{tabular} & \begin{tabular}{|l|}
197 \\
1,05 \\
$+0,07$ \\
\end{tabular} & 0,105 & 0,994 & 0,08 & 0,918 & 0,034 \\
\hline $\begin{array}{l}\text { Magnesium } \\
\text { Excretion, } \\
\mu \mathrm{M} / 24 \mathrm{~h} \cdot 100 \text { g Body Mass }\end{array}$ & $\begin{array}{l}3,30 \\
1 \\
0\end{array}$ & \begin{tabular}{|l|}
3,55 \\
1,07 \\
$+0,12$ \\
\end{tabular} & $\begin{array}{l}4,46 \\
1,35 \\
+0,56\end{array}$ & 0,099 & 0,940 & 0,93 & 0,406 & 0,112 \\
\hline $\begin{array}{l}\text { Chloride } \\
\text { Excretion, } \\
\mu \mathrm{M} / 24 \mathrm{~h} \bullet 100 \mathrm{~g} \text { Body Mass }\end{array}$ & $\begin{array}{l}144 \\
1 \\
0 \\
\end{array}$ & \begin{tabular}{|l|}
107 \\
0,74 \\
$-0,38$ \\
\end{tabular} & \begin{tabular}{|l|}
220 \\
1,52 \\
$+0,76$ \\
\end{tabular} & 0,100 & 0,943 & 0,88 & 0,424 & 0,022 \\
\hline $\begin{array}{l}\text { Calcium } \\
\text { Excretion, } \\
\mu \mathrm{M} / 24 \mathrm{~h} \bullet 100 \mathrm{~g} \text { Body Mass }\end{array}$ & $\begin{array}{l}2,90 \\
1 \\
0\end{array}$ & \begin{tabular}{|l|}
3,22 \\
1,11 \\
$+0,21$ \\
\end{tabular} & $\begin{array}{l}3,86 \\
1,33 \\
+0,63 \\
\end{array}$ & 0,103 & 0,970 & 0,45 & 0,641 & 0,701 \\
\hline $\begin{array}{l}\text { Potassium } \\
\text { Erythrocytes, } \\
\mathrm{mM} / \mathrm{L}\end{array}$ & $\begin{array}{l}87,0 \\
1 \\
0\end{array}$ & \begin{tabular}{|l|}
85,8 \\
0,99 \\
$-0,18$ \\
\end{tabular} & \begin{tabular}{|l|}
87,5 \\
1,01 \\
$+0,08$ \\
\end{tabular} & 0,100 & 0,948 & 0,79 & 0,462 & 0,684 \\
\hline $\begin{array}{l}\text { Sodium } \\
\text { Erythrocytes, } \\
\mathrm{mM} / \mathrm{L}\end{array}$ & $\begin{array}{l}22,0 \\
1 \\
0\end{array}$ & \begin{tabular}{|l|}
22,6 \\
1,03 \\
$+0,13$ \\
\end{tabular} & $\begin{array}{l}23,0 \\
1,05 \\
+0,23 \\
\end{array}$ & 0,104 & 0,986 & 0,20 & 0,817 & 0,453 \\
\hline
\end{tabular}


Table 5. Discriminant Function Analysis Summary. Urine non-electrolytic variables currently not in the model

\begin{tabular}{|c|c|c|c|c|c|c|c|c|}
\hline \multirow[b]{2}{*}{ Variables } & \multicolumn{3}{|c|}{ Groups (n) } & \multicolumn{5}{|c|}{ Parameters of Wilks' Statistics } \\
\hline & $\begin{array}{l}\text { Intact } \\
\text { rats } \\
(10)\end{array}$ & $\begin{array}{l}\text { Daily } \\
\text { Water } \\
\text { (10) }\end{array}$ & $\begin{array}{l}\text { Salt } \\
\text { Waters } \\
\text { (30) }\end{array}$ & $\begin{array}{l}\text { Wilks' } \\
\Lambda\end{array}$ & $\begin{array}{l}\text { Parti- } \\
\text { al } \Lambda\end{array}$ & $\begin{array}{l}\text { F to } \\
\text { en- } \\
\text { ter }\end{array}$ & $\begin{array}{l}\text { p- } \\
\text { level }\end{array}$ & $\begin{array}{l}\text { Tole- } \\
\text { rancy }\end{array}$ \\
\hline $\begin{array}{l}\text { Malondialdehyde } \\
\text { Urine, } \\
\mu \mathrm{M} / \mathrm{L}\end{array}$ & $\begin{array}{l}92 \\
1 \\
0\end{array}$ & $\begin{array}{l}75 \\
0,81 \\
-0,40\end{array}$ & $\begin{array}{l}92 \\
1,00 \\
0,00\end{array}$ & 0,103 & 0,973 & 0,41 & 0,668 & 0,582 \\
\hline $\begin{array}{l}\text { Diene conjugates } \\
\text { Urine, } \\
\mathrm{E}^{232} / \mathrm{mL}\end{array}$ & $\begin{array}{l}1,86 \\
1 \\
0\end{array}$ & $\begin{array}{l}1,68 \\
0,91 \\
-0,26\end{array}$ & $\begin{array}{l}1,87 \\
1,01 \\
+0,03\end{array}$ & 0,102 & 0,966 & 0,51 & 0,605 & 0,527 \\
\hline $\begin{array}{l}\text { Urea } \\
\text { Excretion, } \\
\mu \mathrm{M} / 24 \mathrm{~h} \cdot 100 \mathrm{~g} \text { Body Mass }\end{array}$ & $\begin{array}{l}169 \\
1 \\
0\end{array}$ & $\begin{array}{l}179 \\
1,06 \\
+0,08\end{array}$ & $\begin{array}{l}262 \\
1,55 \\
+0,69\end{array}$ & 0,100 & 0,948 & 0,79 & 0,462 & 0,684 \\
\hline $\begin{array}{l}\text { Urea } \\
\text { Urine, } \\
\mathrm{mM} / \mathrm{L}\end{array}$ & $\begin{array}{l}107 \\
1 \\
0 \\
\end{array}$ & $\begin{array}{l}110 \\
1,03 \\
+0,07 \\
\end{array}$ & $\begin{array}{l}131 \\
1,22 \\
+0,58 \\
\end{array}$ & 0,105 & 0,988 & 0,18 & 0,839 & 0,038 \\
\hline $\begin{array}{l}\text { Uric Acid } \\
\text { Urine, } \\
\mathrm{mM} / \mathrm{L}\end{array}$ & $\begin{array}{l}3,68 \\
1 \\
0\end{array}$ & $\begin{array}{l}4,29 \\
1,17 \\
+0,33 \\
\end{array}$ & $\begin{array}{l}3,30 \\
0,90 \\
-0,20 \\
\end{array}$ & 0,103 & 0,976 & 0,36 & 0,699 & 0,226 \\
\hline $\begin{array}{l}\text { Middle Mass Molecules } \\
\text { Urine, } \\
\text { units }\end{array}$ & $\begin{array}{l}182 \\
1 \\
0 \\
\end{array}$ & $\begin{array}{l}174 \\
0,95 \\
-0,16 \\
\end{array}$ & $\begin{array}{l}158 \\
0,87 \\
-0,46 \\
\end{array}$ & 0,104 & 0,986 & 0,20 & 0,817 & 0,453 \\
\hline $\begin{array}{l}\text { Creatinine } \\
\text { Excretion, } \\
\mu \mathrm{M} / 24 \mathrm{~h} \cdot 100 \mathrm{~g} \text { Body Mass }\end{array}$ & $\begin{array}{l}8,7 \\
1 \\
0 \\
\end{array}$ & $\begin{array}{l}10,7 \\
1,23 \\
+0,46 \\
\end{array}$ & $\begin{array}{l}12,5 \\
1,43 \\
+0,86 \\
\end{array}$ & 0,105 & 0,990 & 0,15 & 0,863 & 0,842 \\
\hline $\begin{array}{l}\text { Creatinine } \\
\text { Urine, } \\
\mathrm{mM} / \mathrm{L}\end{array}$ & $\begin{array}{l}6,41 \\
1 \\
0 \\
\end{array}$ & $\begin{array}{l}7,23 \\
1,13 \\
+0,45 \\
\end{array}$ & $\begin{array}{l}7,16 \\
1,12 \\
+0,41 \\
\end{array}$ & 0,104 & 0,983 & 0,25 & 0,778 & 0,483 \\
\hline $\begin{array}{l}\text { Amylase Activity } \\
\text { Urine, } \\
\mathrm{g} / \mathrm{h} \cdot \mathrm{L}\end{array}$ & $\begin{array}{l}202 \\
1 \\
0 \\
\end{array}$ & $\begin{array}{l}217 \\
1,07 \\
+0,26 \\
\end{array}$ & $\begin{array}{l}204 \\
1,01 \\
+0,03 \\
\end{array}$ & 0,105 & 0,994 & 0,08 & 0,918 & 0,034 \\
\hline $\begin{array}{l}\text { Uric Acid } \\
\text { Excretion, } \\
\mu \mathrm{M} / 24 \mathrm{~h} \cdot 100 \mathrm{~g} \text { Body Mass }\end{array}$ & $\begin{array}{l}5,72 \\
1 \\
0 \\
\end{array}$ & $\begin{array}{l}6,02 \\
1,05 \\
+0,05 \\
\end{array}$ & $\begin{array}{l}5,33 \\
0,93 \\
-0,07 \\
\end{array}$ & 0,100 & 0,948 & 0,79 & 0,462 & 0,684 \\
\hline
\end{tabular}


Table 6. Discriminant Function Analysis Summary. Blood non-electrolytic variables as well as kidney function variables currently not in the model

\begin{tabular}{|c|c|c|c|c|c|c|c|c|}
\hline \multirow[b]{2}{*}{ Variables } & \multicolumn{3}{|c|}{ Groups (n) } & \multicolumn{5}{|c|}{ Parameters of Wilks' Statistics } \\
\hline & $\begin{array}{l}\text { Intact } \\
\text { rats } \\
(10)\end{array}$ & $\begin{array}{l}\text { Daily } \\
\text { Water } \\
\text { (10) }\end{array}$ & $\begin{array}{l}\text { Salt } \\
\text { Waters } \\
(30)\end{array}$ & $\begin{array}{l}\text { Wilks' } \\
\Lambda\end{array}$ & $\begin{array}{l}\text { Parti- } \\
\text { al } \Lambda\end{array}$ & $\begin{array}{l}\text { F to } \\
\text { en- } \\
\text { ter }\end{array}$ & $\begin{array}{l}\mathrm{p}- \\
\text { level }\end{array}$ & $\begin{array}{l}\text { Tole- } \\
\text { rancy }\end{array}$ \\
\hline $\begin{array}{l}\text { Superoxide Dismutase } \\
\text { Erythrocytes, } \\
\text { un/mL }\end{array}$ & $\begin{array}{l}58,0 \\
1 \\
0 \\
\end{array}$ & $\begin{array}{l}58,2 \\
1,00 \\
+0,02 \\
\end{array}$ & $\begin{array}{l}53,8 \\
0,93 \\
-0,39 \\
\end{array}$ & 0,106 & 0,998 & 0,03 & 0,972 & 0,602 \\
\hline $\begin{array}{l}\text { Malondyaldehide } \\
\text { Plasma, } \\
\mu \mathrm{M} / \mathrm{L}\end{array}$ & $\begin{array}{l}63 \\
1 \\
0 \\
\end{array}$ & $\begin{array}{l}79 \\
1,25 \\
+0,74 \\
\end{array}$ & $\begin{array}{l}68 \\
1,08 \\
+0,24 \\
\end{array}$ & 0,105 & 0,992 & 0,11 & 0,896 & 0,205 \\
\hline $\begin{array}{l}\text { Creatinine } \\
\text { Plasma, } \\
\mu \mathrm{M} / \mathrm{L}\end{array}$ & $\begin{array}{l}72,5 \\
1 \\
0 \\
\end{array}$ & $\begin{array}{l}92 \\
1,26 \\
+0,79 \\
\end{array}$ & $\begin{array}{l}76 \\
1,05 \\
+0,14 \\
\end{array}$ & 0,104 & 0,983 & 0,25 & 0,778 & 0,483 \\
\hline $\begin{array}{l}\text { Bilirubin } \\
\text { Plasma, } \\
\mu \mathrm{M} / \mathrm{L} \\
\end{array}$ & $\begin{array}{l}4,63 \\
1 \\
0 \\
\end{array}$ & $\begin{array}{l}4,65 \\
1,00 \\
+0,01 \\
\end{array}$ & $\begin{array}{l}4,34 \\
0,94 \\
-0,11 \\
\end{array}$ & 0,101 & 0,958 & 0,63 & 0,540 & 0,614 \\
\hline $\begin{array}{l}\text { Urea } \\
\text { Plasma, } \\
\mathrm{mM} / \mathrm{L}\end{array}$ & $\begin{array}{l}7,42 \\
1 \\
0 \\
\end{array}$ & $\begin{array}{l}9,46 \\
1,27 \\
+1,19 \\
\end{array}$ & $\begin{array}{l}8,32 \\
1,12 \\
+0,53 \\
\end{array}$ & 0,105 & 0,994 & 0,08 & 0,918 & 0,034 \\
\hline $\begin{array}{l}\text { Middle Mass Molecules } \\
\text { Plasma, } \\
\text { units }\end{array}$ & $\begin{array}{l}154 \\
1 \\
0\end{array}$ & $\begin{array}{l}175 \\
1,14 \\
+0,41\end{array}$ & $\begin{array}{l}129 \\
0,84 \\
-0,48\end{array}$ & 0,099 & 0,940 & 0,93 & 0,406 & 0,112 \\
\hline $\begin{array}{l}\text { Glucose } \\
\text { Plasma, } \\
\mathrm{mM} / \mathrm{L}\end{array}$ & $\begin{array}{l}4,95 \\
1 \\
0\end{array}$ & $\begin{array}{l}5,49 \\
1,11 \\
+0,49\end{array}$ & $\begin{array}{l}5,39 \\
1,09 \\
+0,40\end{array}$ & 0,100 & 0,943 & 0,88 & 0,424 & 0,022 \\
\hline $\begin{array}{l}\text { Amylase Activity } \\
\text { Plasma, } \\
\mathrm{g} / \mathrm{h} \cdot \mathrm{L}\end{array}$ & $\begin{array}{l}152 \\
1 \\
0 \\
\end{array}$ & $\begin{array}{l}154 \\
1,02 \\
+0,10 \\
\end{array}$ & $\begin{array}{l}159 \\
1,05 \\
+0,30 \\
\end{array}$ & 0,103 & 0,970 & 0,45 & 0,641 & 0,701 \\
\hline $\begin{array}{l}\text { Uric Acid } \\
\text { Plasma, } \\
\mu \mathrm{M} / \mathrm{L}\end{array}$ & $\begin{array}{l}662 \\
1 \\
0 \\
\end{array}$ & $\begin{array}{l}620 \\
0,94 \\
-0,12 \\
\end{array}$ & $\begin{array}{l}787 \\
1,19 \\
+0,37 \\
\end{array}$ & 0,105 & 0,988 & 0,18 & 0,839 & 0,038 \\
\hline $\begin{array}{l}\text { Diuresis, } \\
\text { mL/24h•100 g Body Mass }\end{array}$ & $\begin{array}{l}1,44 \\
1 \\
0\end{array}$ & $\begin{array}{l}1,48 \\
1,03 \\
+0,05\end{array}$ & $\begin{array}{l}1,83 \\
1,27 \\
+0,43\end{array}$ & 0,103 & 0,976 & 0,36 & 0,699 & 0,226 \\
\hline $\begin{array}{l}\text { Canalicular } \\
\text { Reabsorbtion, } \\
\%\end{array}$ & $\begin{array}{l}98,7 \\
1 \\
0 \\
\end{array}$ & $\begin{array}{l}98,6 \\
1,00 \\
-0,05 \\
\end{array}$ & $\begin{array}{l}98,9 \\
1,00 \\
+0,23 \\
\end{array}$ & 0,104 & 0,986 & 0,20 & 0,817 & 0,453 \\
\hline $\begin{array}{l}(\mathrm{Ca} \cdot \mathrm{UA} / \mathrm{Mg} \cdot \mathrm{Cr})^{0,25} \\
\text { as Lithogenicity } \\
\text { Urine Index }\end{array}$ & $\begin{array}{l}0,90 \\
1 \\
0\end{array}$ & $\begin{array}{l}0,90 \\
1,00 \\
0,00\end{array}$ & $\begin{array}{l}0,82 \\
0,91 \\
-0,31\end{array}$ & 0,100 & 0,948 & 0,79 & 0,462 & 0,684 \\
\hline
\end{tabular}


Table 7. Summary of Stepwise Analysis

\begin{tabular}{|l|l|l|l|l|l|}
\hline $\begin{array}{l}\text { Variables } \\
\text { currently in the model }\end{array}$ & $\begin{array}{l}\text { F to } \\
\text { enter }\end{array}$ & $\begin{array}{l}\text { p- } \\
\text { level }\end{array}$ & $\Lambda$ & $\begin{array}{l}\text { F- } \\
\text { value }\end{array}$ & $\begin{array}{l}\text { p- } \\
\text { level }\end{array}$ \\
\hline Calcium Plasma & 5,92 & 0,005 & 0,799 & 5,92 & 0,005 \\
\hline Potassium Plasma & 4,33 & 0,019 & 0,672 & 5,05 & 0,001 \\
\hline Sodium Excretion & 5,34 & 0,008 & 0,543 & 5,35 & $10^{-4}$ \\
\hline (Cap/Pp) $^{\mathbf{0}, 5}$ as Parathyroid Activity & 4,43 & 0,018 & 0,452 & 5,36 & $10^{-4}$ \\
\hline Glomerular Filtration & 3,21 & 0,050 & 0,393 & 5,11 & $10^{-5}$ \\
\hline Glomerular Zone of Adrenal Cortex & 1,93 & 0,157 & 0,360 & 4,66 & $10^{-5}$ \\
\hline Katalase Plasma & 1,94 & 0,157 & 0,329 & 4,35 & $10^{-5}$ \\
\hline Mode HRV as Humoral channel & 2,45 & 0,099 & 0,293 & 4,24 & $10^{-5}$ \\
\hline Diene conjugates Plasma & 2,68 & 0,081 & 0,258 & 4,20 & $10^{-5}$ \\
\hline Sodium Plasma & 1,86 & 0,169 & 0,235 & 4,04 & $10^{-5}$ \\
\hline Cholesterol Plasma & 2,13 & 0,133 & 0,210 & 3,97 & $10^{-5}$ \\
\hline Medullar Zone of Adrenals & 2,27 & 0,118 & 0,187 & 3,94 & $10^{-5}$ \\
\hline Triiodothyronine Plasma & 1,37 & 0,268 & 0,173 & 3,78 & $10^{-5}$ \\
\hline Phosphate Plasma & 2,12 & 0,136 & 0,154 & 3,76 & $10^{-5}$ \\
\hline Chloride Plasma & 1,74 & 0,192 & 0,139 & 3,69 & $10^{-5}$ \\
\hline Katalase Urine & 2,16 & 0,131 & 0,123 & 3,71 & $10^{-5}$ \\
\hline Testosterone Plasma & 1,28 & 0,294 & 0,113 & 3,59 & $10^{-5}$ \\
\hline Magnesium Plasma & 1,09 & 0,351 & 0,106 & 3,46 & $10^{-5}$ \\
\hline
\end{tabular}

The dividing information contained in 18 variables is condensed in 2 canonical discriminant roots (Table 8 ). The major root contains $68,7 \%$ of discriminative opportunities $\left(\mathrm{r}^{*}=0,866\right.$; Wilks' $\left.\Lambda=0,1058 ; \chi^{2}{ }_{(36)}=86 ; \mathrm{p}<10^{-5}\right)$ and the minor root $31,3 \%\left(\mathrm{r}^{*}=0,760\right.$; Wilks' $\left.\Lambda=0,4227 ; \chi^{2}(17)=33 ; \mathrm{p}=0,011\right)$.

Table 8 shows standardized (normalized) and non-standardized (raw) coefficients for discriminant variables. The calculation of the discriminant root values for each animal as the sum of the products of raw coefficients to the individual values of discriminant variables together with the constant enables the visualization of each rat in the information space of the roots (Fig. 1).

Table 8. Standardized and Raw Coefficients for Canonical Variables

\begin{tabular}{|l|l|l|l|l|}
\hline \multicolumn{1}{|c|}{ Coefficients } & \multicolumn{2}{|c|}{ Standardized } & \multicolumn{2}{c|}{ Raw } \\
\hline Variables & Root 1 & Root 2 & Root 1 & Root 2 \\
\hline Calcium Plasma & $-0,209$ & $-0,292$ & $-0,254$ & $-0,355$ \\
\hline Potassium Plasma & 0,967 & 0,616 & 1,277 & 0,813 \\
\hline Sodium Excretion & $-0,494$ & $-0,413$ & $-0,0028$ & $-0,0024$ \\
\hline (Cap/Pp),5 as Parathyroid Activity & 1,685 & 0,567 & 2,468 & 0,830 \\
\hline Glomerular Filtration & $-0,121$ & $-0,449$ & $-0,0014$ & $-0,0053$ \\
\hline Glomerular Zone of Adrenal Cortex & 0,474 & 0,364 & 0,013 & 0,010 \\
\hline Katalase Plasma & $-1,484$ & 0,187 & $-0,032$ & 0,004 \\
\hline Mode HRV as Humoral channel & 0,812 & $-0,0004$ & 0,0412 & $-0,00002$ \\
\hline Diene conjugates Plasma & $-0,996$ & $-0,035$ & $-2,176$ & $-0,076$ \\
\hline Sodium Plasma & $-1,856$ & 1,139 & $-0,346$ & 0,212 \\
\hline Cholesterol Plasma & $-0,276$ & 0,341 & $-0,650$ & 0,803 \\
\hline Medullar Zone of Adrenals & 0,439 & $-0,662$ & 0,013 & $-0,020$ \\
\hline Triiodothyronine Plasma & $-0,112$ & $-0,656$ & $-0,276$ & $-1,618$ \\
\hline Phosphate Plasma & 1,506 & 0,105 & 2,784 & 0,194 \\
\hline Chloride Plasma & 1,368 & $-0,946$ & 0,214 & $-0,148$ \\
\hline Katalase Urine & 1,221 & 0,039 & 0,031 & 0,001 \\
\hline Testosterone Plasma & $-0,292$ & 0,340 & $-0,142$ & 0,166 \\
\hline Magnesium Plasma & 0,228 & 0,465 & 0,452 & 0,921 \\
\hline & \multicolumn{2}{|c|}{ Constants } & 10,41 & $-16,16$ \\
\hline
\end{tabular}




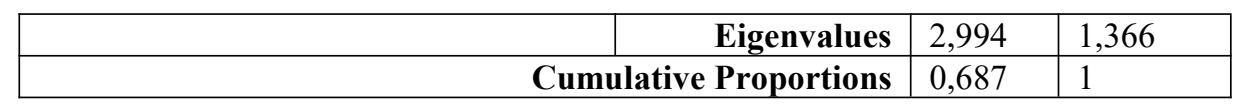

In the Table 9 together with discriminant variables are also variables that carry identifying/ separating information, but were outside the model due to its duplication/redundancy. For ease of comparison, the values of the variables are transformed into Z-scores.

The localization of the cluster of control rats in the extreme left zone of the first root axis (Fig. 1) reflects their maximally elevated levels of testosterone, circulating catechol amines, sympathetic tone and mineralocorticoid activity, on the one hand, and maximally reduced levels of parathyroid activity, corticosteronemia, vagal tone and thickness of medullar zone of adrenals.

Since control rats received the same water as intact, but through a metal tube with prefixation in the experimenter's hand, the detected changes in neuroendocrine status, apparently, is a manifestation of chronic aversive stress [16-20, 23,25,27].

Metabolic manifestations of chronic stress, apparently, are increased plasma levels of urea, creatinine and malondialdehyde as well as catalase activity in plasma and urine [27].

The tested mineral waters minimize or eliminate the neuroendocrine and metabolic manifestations of chronic stress, ie have a stress-limiting effect. 
Table 9. Factor Structure Matrix (Correlations Variables-Canonical Roots) and Means of Roots and Variables

\begin{tabular}{|c|c|c|c|c|c|}
\hline \multirow[b]{2}{*}{ Root $1(68,7 \%)$} & \multicolumn{2}{|c|}{$\begin{array}{c}\text { Correlations } \\
\text { Variables-Roots }\end{array}$} & \multirow{2}{*}{$\begin{array}{l}\text { Daily } \\
\text { Water } \\
-1,73\end{array}$} & \multirow{2}{*}{$\begin{array}{l}\begin{array}{l}\text { Salt } \\
\text { Waters }\end{array} \\
-0,50\end{array}$} & \multirow{2}{*}{$\begin{array}{l}\begin{array}{l}\text { Intact } \\
\text { rats }\end{array} \\
+3,21 \\
\end{array}$} \\
\hline & R1 & $\mathbf{R 2}$ & & & \\
\hline Testosterone Plasma & $-0,169$ & 0,148 & $+1,97$ & $+0,77$ & 0 \\
\hline 1/Mode as Circulating Catechol amines & $-0,150$ & 0,169 & $+1,27$ & $+0,34$ & 0 \\
\hline Amplitude Mode as Sympathetic tone & & & $+0,84$ & $-0,01$ & 0 \\
\hline$(\mathrm{Ku} / \mathrm{Nau})^{0,5}$ as Mineralocorticoid Activity & & & $+1,09$ & $-0,05$ & 0 \\
\hline Katalase Plasma Activity & $-0,162$ & 0,086 & $+1,58$ & $+0,77$ & 0 \\
\hline Katalase Urine Activity & $-0,137$ & $-0,038$ & $+0,96$ & $+0,86$ & 0 \\
\hline Malondyaldehide Plasma & & & $+0,74$ & $+0,24$ & 0 \\
\hline Urea Plasma & & & $+1,19$ & $+0,53$ & 0 \\
\hline Creatinine Plasma & & & $+0,79$ & $+0,14$ & 0 \\
\hline Phosphate Plasma & $-0,122$ & $-0,043$ & $+0,65$ & $+0,53$ & 0 \\
\hline Calcium Plasma & 0,268 & $-0,164$ & $-1,24$ & $-0,63$ & 0 \\
\hline$(\mathrm{Cap} / \mathrm{Pp})^{0,5}$ as Parathyroid Activity & 0,289 & $-0,003$ & $-0,84$ & $-0,63$ & 0 \\
\hline Medullar Zone of Adrenals & 0,099 & $-0,241$ & $-0,93$ & $-0,01$ & 0 \\
\hline Corticosterone Plasma & & & $-0,78$ & $-0,55$ & 0 \\
\hline MxDMn HRV as Vagal tone & & & $-0,39$ & $+0,05$ & 0 \\
\hline Root $2(31,3 \%)$ & R1 & $\mathbf{R 2}$ & $+1,94$ & $-0,86$ & $+0,65$ \\
\hline Triiodothyronine Plasma & $-0,044$ & $-0,229$ & $-0,05$ & $+0,36$ & 0 \\
\hline Fascicular Zone of Adrenal Cortex & & & $+0,09$ & $+0,34$ & 0 \\
\hline Glomerular Filtration & $-0,074$ & $-0,291$ & $-0,03$ & $+1,97$ & 0 \\
\hline Diuresis & & & $+0,05$ & $+0,43$ & 0 \\
\hline Sodium Excretion & $-0,010$ & $-0,297$ & $-0,70$ & $+1,00$ & 0 \\
\hline Chloride Excretion & & & $-0,38$ & $+0,76$ & 0 \\
\hline Calcium Excretion & & & $+0,21$ & $+0,63$ & 0 \\
\hline Magnesium Excretion & & & $+0,12$ & $+0,56$ & 0 \\
\hline Creatinine Excretion & & & $+0,46$ & $+0,86$ & 0 \\
\hline Urea Excretion & & & $+0,08$ & $+0,69$ & 0 \\
\hline Urea Urine & & & $+0,07$ & $+\mathbf{0 , 5 8}$ & \\
\hline Phosphates Excretion & & & $+0,08$ & $+0,33$ & 0 \\
\hline Sodium Urine & & & $-0,76$ & $+0,32$ & 0 \\
\hline Chloride Urine & & & $-0,56$ & $+0,28$ & 0 \\
\hline Diene conjugates Plasma & $-0,059$ & $-0,090$ & $+0,20$ & $+\mathbf{0 , 3 9}$ & 0 \\
\hline Uric Acid Plasma & & & $-0,12$ & $+\mathbf{0 , 3 7}$ & 0 \\
\hline Glomerular Zone of Adrenal Cortex & $-0,019$ & 0,220 & $+0,29$ & $-0,21$ & 0 \\
\hline Potassium Plasma & 0,231 & 0,178 & $-0,98$ & $-1,21$ & 0 \\
\hline Chloride Plasma & 0,040 & 0,278 & $+0,14$ & $-0,51$ & 0 \\
\hline Sodium Plasma & $-0,060$ & 0,251 & $+0,65$ & $-0,16$ & 0 \\
\hline Magnesium Plasma & $-0,000$ & 0,167 & $+0,19$ & $-0,24$ & 0 \\
\hline Middle Mass Molecules Plasmas & & & $+0,41$ & $-0,48$ & 0 \\
\hline Middle Mass Molecules Urine & & & $-0,16$ & $-0,46$ & 0 \\
\hline Uric Acid Urine & & & $+0,33$ & $-0,20$ & 0 \\
\hline$(\mathrm{Ca} \cdot \mathrm{UA} / \mathrm{Mg} \cdot \mathrm{Cr})^{0,25}$ as Urolithogenicity & & & 0,00 & $-0,31$ & 0 \\
\hline Superoxide Dismutase Erythrocytes & & & $+0,02$ & $-0,39$ & 0 \\
\hline Cholesterol Plasma & $-0,033$ & 0,108 & $+0,28$ & $-0,01$ & 0 \\
\hline
\end{tabular}

The stress-limiting effect of mineral waters is illustrated by the shift of the localization of their cluster towards the cluster of intact animals. However, the distinction with control animals is not entirely clear. Additional delimitation occurs along the axis of the second root. The lowest location of mineral-loaded rat points reflects the maximum sampling level of triiodothyronine and the thickness of the fascicular layer of the adrenal cortex combined with 
maxima of glomerular filtration, diuresis and excretion of electrolytes and nitrogenous metabolites, as well as plasma levels of diene conjugates and uric acid. On the other hand, these rats are characterized by the minimum thickness of the glomerular layer of the adrenal cortex and the minimum plasma levels of electrolytes regulated by its hormones, as well as the activity of superoxide dismutase of erythrocytes and molecules of medium mass. The level of the latter is minimal also in urine, as well as uric acid and lithogenicity of urine.

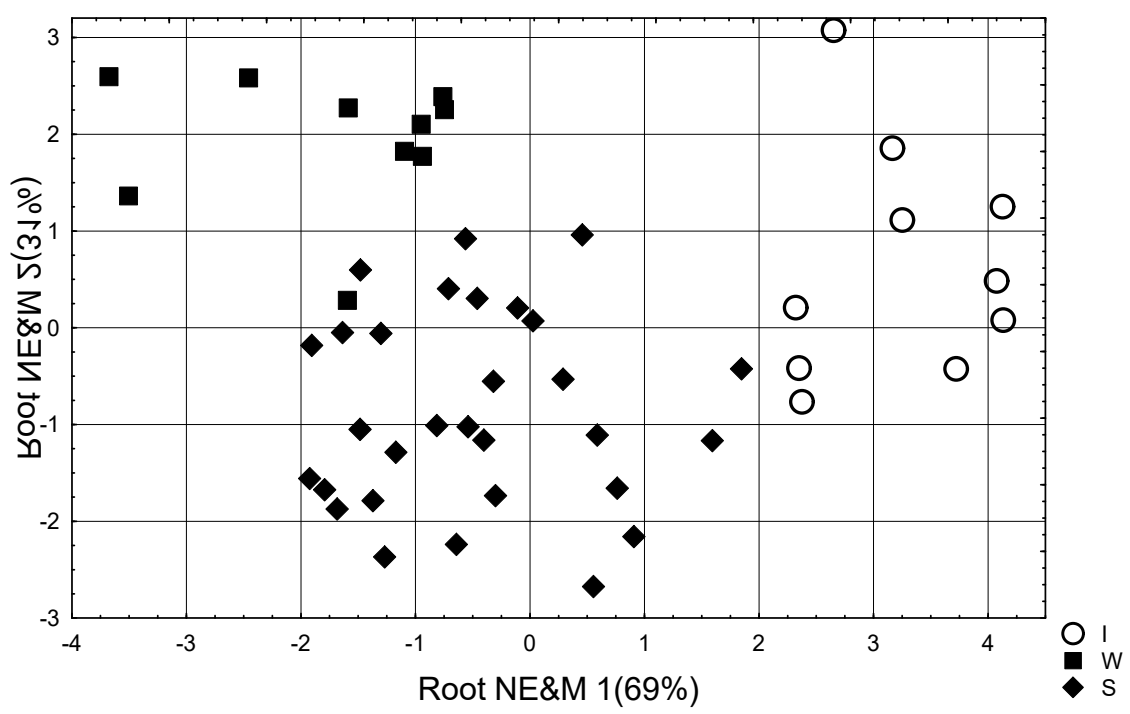

Fig. 1. Individual values of the first and second roots of the neuroendocrine and metabolic parameters in intact rats (I) and loaded with Daily water (W) and Salt waters (S)

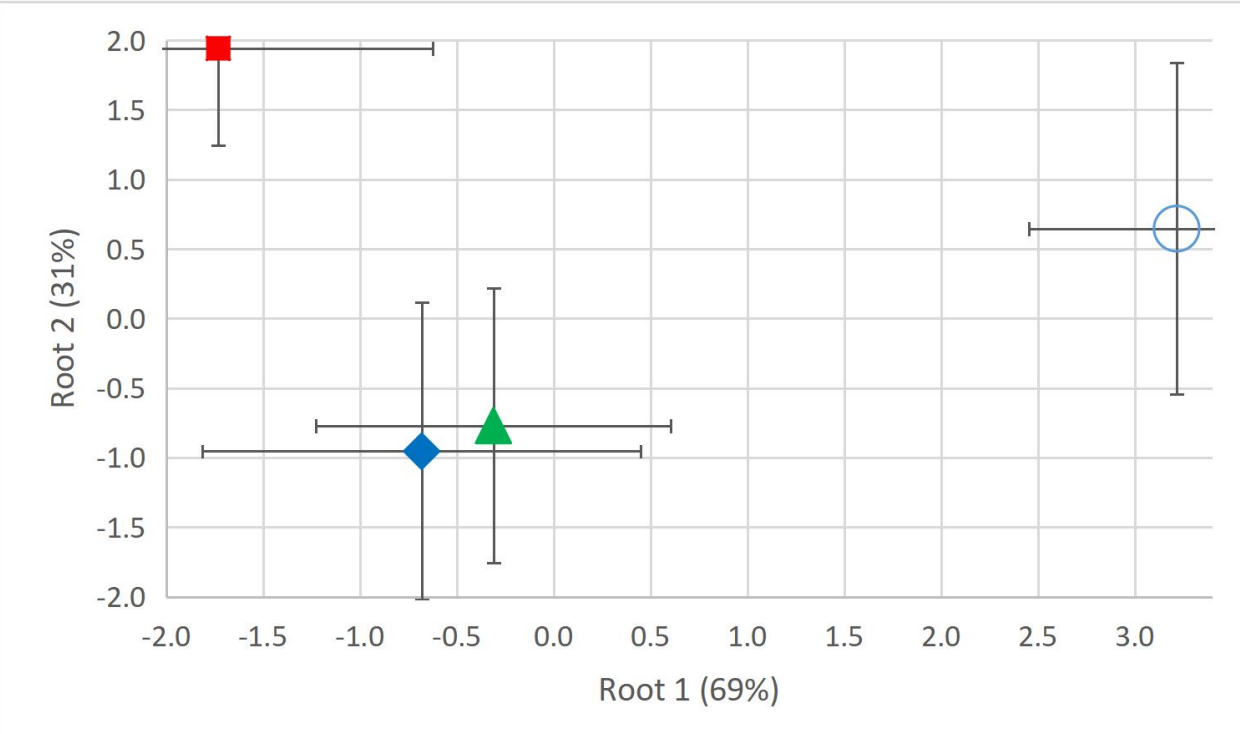

Fig. 2. Average values $(M e a n \pm S D)$ of the first and second roots of the neuroendocrine and metabolic parameters in intact rats $(\mathrm{O})$ and loaded with Daily water and Salt waters Myroslava or Khrystyna

Figure 2 illustrates the lack of differences between the two mineral waters in the set of discriminant variables.

On the whole, in the information space of the discriminating roots, all groups are clearly delineated, that is, they differ from each other by constellation of 18 metabolic and 
neuroendocrine parameters. This distinction is documented by calculating the squared Mahalanobis distances between them (Table 10).

Table 10. Squared Mahalanobis Distances between groups (over diagonal), F-values $(\mathrm{df}=18,3)$ and $p$-levels (under diagonal)

\begin{tabular}{|l|l|l|l|}
\hline Groups & $\begin{array}{l}\text { I } \\
(10)\end{array}$ & $\begin{array}{l}\text { DW } \\
(\mathbf{1 0})\end{array}$ & $\begin{array}{l}\text { SW } \\
(\mathbf{3 0})\end{array}$ \\
\hline Intact rats (I) & $\mathbf{0 , 0}$ & 26,1 & 16,1 \\
\hline $\begin{array}{l}\text { Daily Water } \\
\text { (DW) }\end{array}$ & $\mathbf{4 , 6 4}$ & $\mathbf{0 , 0}$ & 9,4 \\
\hline Salt Waters & $\mathbf{, 0 0 0 1}$ & & \\
(SW) & $\mathbf{4 , 2 7}$ & $\mathbf{2 , 5 0}$ & $\mathbf{0 , 0}$ \\
\hline
\end{tabular}

The application of the classifying functions (Table 11) enables the retrospective identification of intact rats unmistakable, and the other two groups - with a single error (Table 12).

Table 11. Coefficients and Constants for Classification Functions

\begin{tabular}{|l|l|l|l|}
\hline Variables currently in the model & $\begin{array}{l}\text { Intact } \\
\text { rats }\end{array}$ & $\begin{array}{l}\text { Daily } \\
\text { Water }\end{array}$ & $\begin{array}{l}\text { Salt } \\
\text { Waters }\end{array}$ \\
\hline Calcium Plasma & 1,778 & 2,573 & 3,254 \\
\hline Potassium Plasma & 19,33 & 14,07 & 13,36 \\
\hline Sodium Excretion & $-0,014$ & $-0,0032$ & 0,0002 \\
\hline (Cap/Pp) $^{\mathbf{0} 5}$ as Parathyroid Activity & $-7,293$ & $-18,43$ & $-17,71$ \\
\hline Glomerular Filtration & $-0,237$ & $-0,236$ & $-0,223$ \\
\hline Glomerular Zone of Adrenal Cortex & 0,237 & 0,185 & 0,172 \\
\hline Katalase Activity Plasma & $-0,066$ & 0,098 & 0,047 \\
\hline Mode HRV as Humoral channel & 0,914 & 0,711 & 0,761 \\
\hline Diene conjugates Plasma & 7,478 & 18,14 & 15,67 \\
\hline Sodium Plasma & 24,44 & 26,42 & 25,40 \\
\hline Cholesterol Plasma & 26,42 & 30,67 & 27,62 \\
\hline Medullar Zone of Adrenals & $-0,240$ & $-0,333$ & $-0,259$ \\
\hline Triiodothyronine Plasma & 11,88 & 11,15 & 15,34 \\
\hline Phosphate Plasma & $-41,86$ & $-55,38$ & $-52,48$ \\
\hline Chloride Plasma & $-16,73$ & $-17,98$ & $-17,30$ \\
\hline Katalase Activity Urine & 0,393 & 0,241 & 0,277 \\
\hline Testosterone Plasma & 2,848 & 3,767 & 3,127 \\
\hline Magnesium Plasma & 38,08 & 37,04 & 35,02 \\
\hline Constants & $-941,7$ & -1012 & $-949,9$ \\
\hline
\end{tabular}

Table 12. Classification Matrix

Rows: Observed classifications; Columns: Predicted classifications

\begin{tabular}{|c|c|c|c|c|}
\hline \multirow[b]{2}{*}{ Groups } & \multirow{2}{*}{$\begin{array}{l}\text { Percent } \\
\text { correct }\end{array}$} & $\mathrm{I}$ & DW & SW \\
\hline & & $\mathrm{p}=, 20$ & $\mathrm{p}=, 20$ & $\mathrm{p}=, 60$ \\
\hline Intact rats (I) & 100 & 10 & 0 & 0 \\
\hline Daily Water (DW) & 90,0 & 0 & 9 & 1 \\
\hline Salt Waters (SW) & 96,7 & 1 & 0 & 29 \\
\hline Total & 96,0 & 11 & 9 & 30 \\
\hline
\end{tabular}

\section{CONCLUSION}


The newly created sulfate-chloride sodium-magnesium drinking mineral waters of Truskavets' spa have similar neuroendocrine and metabolic effects on healthy old female rats significantly different from daily water.

\section{CONFORMITY TO ETHICAL STANDARDS}

Experiments on animals have been carried out in accordance with the provisions of the Helsinki Declaration of 1975, revised and supplemented in 2002 by the Directives of the National Committees for Ethics in Scientific Research.

The conduct of experiments was approved by the Ethics Committee of the Horbachevskyi Ternopil' National Medical University. The modern rules for the maintenance and use of laboratory animals complying with the principles of the European Convention for the Protection of Vertebrate Animals used for scientific experiments and needs are observed (Strasbourg, 1985).

\section{REFERENCES}

1. Andreyeva LI, Kozhemyakin LA, Kishkun AA. Modification of the method for determining the lipid peroxide in the test with thiobarbituric acid [in Russian]. Laboratornoye Delo. 1988; 11: 4143.

2. Baevskiy RM, Kirillov OI, Kletskin SZ. Mathematical Analysis of Changes in Heart Rate by Stress [in Russian]. Moskva. Nauka; 1984: 221.

3. Bilas VR, Popovych IL. Role of microflora and organic substances of water Naftussya in its modulating influence on neuroendocrine-immune complex and metabolism [in Ukrainian]. Medical Hydrology and Rehabilitation. 2009; 7(1): 68-102.

4. Dubinina YY, Yefimova LF, Sofronova LN, Geronimus AL. Comparative analysis of the activity of superoxide dismutase and catalase of erythrocytes and whole blood from newborn children with chronic hypoxia [in Russian]. Laboratornoye Delo. 1988; 8: 16-19.

5. Flyunt VR, Flyunt I-SS, Fil' VM, Kovbasnyuk MM, Hryvnak RF, Popel SL, Zukow W. Relationships between caused by drinking of bioactive water Naftussya changes in urine lithogenicity and neuro-humoral-immune factors in humans with their abnormalities. Journal of Education, Health and Sport. 2017; 7(3): 11-30.

6. Gavrilov VB, Mishkorudnaya MI. Spectrophotometric determination of plasma levels of lipid hydroperoxides [in Russian]. Laboratornoye Delo. 1983; 3: 33-36.

7. Goryachkovskiy AM. Clinical Biochemistry [in Russian]. Odesa: Astroprint; 1998: 608.

8. Gozhenko AI. Functional-metabolic continuum [in Russian]. J of NAMS of Ukraine. 2016; 22 (1): $3-8$.

9. Hrytsak MV, Barylyak LG, Usyns'kyi RS, Mysula IR. Endocrine and metabolic effects of sulfate chloride sodium-magnesium mineral waters "Myroslava" and "Khrystyna" of Truskavets' spa in healthy female rats. In: Proceedings of the XII All-Ukrainian scientific-practical conference "Topical issues of pathology under the influence of extraordinary factors on the body". Galician Readings II (Ternopil', October 29-30, 2020). Ternopil'; 2020: 125-127.

10. Hrytsan II, Gozhenko AI, Badiuk NS, Zukow W. Variants of the state of electrolyte exchange in female rates. In: Rehabilitation Medicine and Health-Resort Institutions Development. Proceedings of the 19th International Applied Research Conference (Kyïv, 11-12 December 2019). Edited by O. Gozhenko, W. Zukow. Toruń, Kyiv. 2019: 25-26.

11. Hrytsan II, Gozhenko AI, Badiuk NS, Zukow W. Variants of the state of electrolyte exchange in female rates. Journal of Education, Health and Sport. 2019; 9(10): 262-279.

12. Klecka WR. Discriminant Analysis [trans. from English in Russian] (Seventh Printing, 1986). In: Factor, Discriminant and Cluster Analysis. Moskva: Finansy i Statistika; 1989: 78-138.

13. Korolyuk MA, Ivanova MI, Mayorova IG, Tokarev VYe. The method for determining the activity of catalase [in Russian]. Laboratornoye Delo. 1988; 1: 16-19. 
14. Kozyavkina OV, Kozyavkina NV, Gozhenko OA, Gozhenko AI, Barylyak LG., Popovych IL. Bioactive Water Naftussya and Neuro-Endocrine-Immune Complex [in Ukrainian]. Kyiv: UNESCOSOCIO; 2015: 349.

15. Makarenko YeV. A comprehensive definition of the activity of superoxide dismutase and glutathione reductase in red blood cells in patients with chronic liver disease [in Russian]. Laboratornoye Delo. 1988; 11: 48-50.

16. Polovynko IS. Integrated quantitative estimation of neuro-endocrine manifestations of chronic stress in female rats. Experimental and Clinical Physiology and Biochemistry. 2017; 3(79): 5-10.

17. Polovynko IS, Zajats LM, Popovych AI, Popovych IL. Integral quantitative evaluation of neuroendocrine and immune reactions to chronic stress in male rats [in Ukrainian]. In: Pathophysiology and Pharmacy: ways of integration: Abstracts of the VII National Congress of Pathophysiologists of Ukraine with International Participation (October 5-7, 2016). Kharkiv: Nat Pharmac Univer: 182.

18. Polovynko IS, Zayats LM, Zukow W, Popovych IL. Neuro-endocrine-immune relationships by chronic stress at male rats. Journal of Health Sciences. 2013; 3(12): 365-374.

19. Polovynko IS, Zajats LM, Zukow W, Yanchij RI, Popovych IL. Quantitative evaluation of integrated neuroendocrine and immune responses to chronic stress in rats male. Journal of Education, Health and Sport. 2016; 6(8): 154-166.

20. Polovynko IS, Zukow W. Variety of immune responses to chronic stress in rats male. Journal of Education, Health and Sport. 2016; 6(12): 843-856.

21. Popovych IL. Functional interactions between neuroendocrine-immune complex in males rats [in Ukrainian]. Achievements of Clinical and Experimental Medicine. 2008; 2(9): 80-87.

22. Popovych IL. The concept of neuroendocrine-immune complex (Review) [in Russian]. Medical Hydrology and Rehabilitation. 2009; 7(3): 9-18

23. Popovych IL, Gozhenko AI, Zukow W, Polovynko IS. Variety of Immune Responses to Chronic Stress and their Neuro-Endocrine Accompaniment. Scholars' Press. Riga; 2020: 172.

24. Tiselius HS. A biochemical basis for grouping of patients with urolithiasis. Europ Urol. 1978; 4: 241-249.

25. Zajats LM, Polovynko IS, Zukow W. Features neuro-endocrine support diversity of immune responses to chronic stress in male rats. Journal of Education, Health and Sport. 2017; 7(3): 97-105.

26. Zajats LM, Polovynko IS, Zukow W, Yanchij RI, Mysakovets' OG, Mel'nyk OI, Hrytsak YaL. Neuroendocrine-immune relatioships in rats females. Journal of Education, Health and Sport. 2017; 7(10): 59-78.

27. Zavidnyuk YV, Mysula IR, Klishch IM, Zukow W, Popovych IL, Korda MM. General nonspecific metabolic, neuroendocrine and immune reactions to various water-salt loads in female rats. Journal of Education, Health and Sport. 2018; 8(3): 513-524. 